\title{
On the use of fluorescence probes for detecting reactive oxygen and nitrogen species associated with photodynamic therapy
}

\author{
Michael Price \\ Wayne State University School of Medicine \\ Cancer Biology Program \\ Detroit, Michigan, 48201

\section{David Kessel} \\ Wayne State University School of Medicine \\ Cancer Biology Program \\ and \\ Department of Pharmacology \\ Detroit, Michigan, 48201
}

\begin{abstract}
Fluorescent probes are frequently employed for the detection of different reactive oxygen and nitrogen species formed during the irradiation of photosensitized cells and tissues. Investigators often interpret the results in terms of information provided with the different probes without examining specificity or determinants of fluorogenic reactions. We examine five fluorescent probes in a cell-free system: reduced 2', $7^{\prime}$-dichlorofluorescein, dihydroethidine, dihydrorhodamine, $3^{\prime}-(p$ aminophenyl) fluorescein (APF), and $4^{\prime}, 5^{\prime}$-diaminofluorescein. Of these, only APF demonstrates a high degree of specificity for a single reactive species. There is a substantial influence of peroxidase activity on all fluorogenic interactions. The fluorescence of the photosensitizing agent also must be taken into account in evaluating results. ๑) 2010 Society of Photo-Optical Instrumentation Engineers. [DOI: 10.1117/1.3484258]
\end{abstract}

Keywords: fluorescent probes; reactive oxygen species; reactive nitrogen species; photodynamic.

Paper 10024SSR received Jan. 17, 2010; revised manuscript received May 13, 2010; accepted for publication May 26, 2010; published online Sep. 2, 2010.
The literature contains numerous reports on the use of fluorescent probes for detection of reactive oxygen (ROS) and nitrogen (RNS) species. Invitrogen/Molecular Probes provides a collection of such probes for which the only indication of specificity is provided by the reproduction of a table derived from Ref. 1. This compares the fluorescence of two new fluorescein analogs, APF and 3'-(p-hydroxyphenyl) fluorescein (HPF), with the fluorescence of DCF in a cell-free system containing different reagents.

In the present study, we examined fluorogenic reactions associated with the exposure of five fluorogenic probes to reactive oxygen and nitrogen species, also in a cell-free system, following procedures reported in Ref. 1. The species examined were $\cdot \mathrm{O}_{2}^{-}\left[100 \mu \mathrm{M} \mathrm{KO}_{2}\right.$ in anhydrous DMSO $], \mathrm{H}_{2} \mathrm{O}_{2}$ $(100 \mu \mathrm{M})$, and $\cdot \mathrm{OH}$ [formed from $20 \mu \mathrm{M} \mathrm{Fe}\left(\mathrm{NH}_{4}\right) \mathrm{SO}_{4}$ $+100 \mu \mathrm{M} \mathrm{H}_{2} \mathrm{O}_{2}$ ]. Fluorogenic effects of reactive nitrogen species were estimated using diethylamine nitric oxide [DEANO, $100 \mu \mathrm{M}$ ]. In aqueous media, NO is released from this compound at $\mathrm{pH}$ 7. NO is then spontaneously oxidized to the nitrosonium cation.

Tests were carried out using $5 \mu \mathrm{M}$ concentrations of each probe in $3 \mathrm{ml}$ of HEPES buffer $\mathrm{pH}$ 7. Fluorescence was measured $30 \mathrm{~min}$ after addition of the reagents specified above. Fluorescence excitation was provided by a 100-W quartzhalogen lamp with the wavelength selected by a monochromator. The fluorescence signal was monitored using an Instaspec IV (Oriel Corp, Stratford, Connecticut) CCD system. Excitation wavelengths were $485 \mathrm{~nm} \quad\left[4^{\prime}, 5^{\prime}\right.$-diamino-

Address all correspondence to David Kessel, Wayne State University, Department of Pharmacology, 540 East Canfield, Detroit, Michigan, 48201, United States of America.Tel: 313577 1766,E-Mail: hkessel@med.wayne.edu fluorescein $\quad(\mathrm{DAF})], \quad 500 \mathrm{~nm} \quad\left[2^{\prime}, 7^{\prime}\right.$-dichlorofluorescein (DCF) and dihydroethidine (DHE)], and $490 \mathrm{~nm}$ [dihydrorhodamine (DHR) and 3' ( $p$-aminophenyl) fluorescein (APF)]. The fluorescence intensity at the emission optimum was recorded. All probes were obtained from Invitrogen/Molecular Probes, Eugene, Oregon, except for DEANO (Cayman Chemical Co., Ann Arbor, Michigan). Horseradish peroxide (HRP, $50 \mu \mathrm{g} / \mathrm{ml}$ ) was present where specified. In studies involving DHE, DNA $(50 \mu \mathrm{g} / \mathrm{ml})$ was added since the longwavelength fluorescence signal depends on binding of the oxidation product(s) to DNA.

Results are summarized in Table 1. The fluorogenic response by DCF was elicited by $\cdot \mathrm{OH} \gg \mathrm{H}_{2} \mathrm{O}_{2}>\cdot \mathrm{O}_{2}^{-}$, with enhanced promotion when peroxidase was present. DHE and DHR also responded to these ROS, but no substantial degree of selectivity for any ROS was observed. It has been reported that $\mathrm{DHE}$ can be selective for $\cdot \mathrm{O}_{2}^{-}$detection if fluorescence (in the presence of DNA) is monitored ${ }^{2}$ at $570 \mathrm{~nm}$, but this probe cannot be used for an unambiguous detection of superoxide without an HPLC analysis of products. ${ }^{3}$ The presence of HRP also led to a strong promotion of probe fluorescence. HRP can promote probe oxidation by a variety of mechanisms including by direct interactions and via conversion of $\mathrm{H}_{2} \mathrm{O}_{2}$ to $\cdot \mathrm{O}_{2}^{-}$ and $\cdot \mathrm{OH} .{ }^{4,5}$ While APF was selective for $\cdot \mathrm{OH}$, especially in the presence of HPR, we have reported ${ }^{6}$ that this probe can also detect ${ }^{1} \mathrm{O}_{2}$ to a greater extent than was suggested by Ref. 1. DAF was converted to a fluorescent product $\mathrm{NO}>\cdot \mathrm{OH}$ $\gg \mathrm{H}_{2} \mathrm{O}_{2}$ and $\cdot \mathrm{O}_{2}^{-}$; there was also an increase in fluorescence when HRP was present. It has been reported ${ }^{1}$ that APF can

1083-3668/2010/15(5)/051605/3/\$25.00 @ 2010 SPIE 
Table 1 Probe:ROS interactions.

\begin{tabular}{|c|c|c|c|c|c|c|c|c|}
\hline \multirow[b]{2}{*}{ Probe } & \multicolumn{2}{|c|}{$\mathrm{H}_{2} \mathrm{O}_{2}$} & \multicolumn{2}{|c|}{$\cdot \mathrm{OH}$} & \multicolumn{2}{|c|}{$\cdot \mathrm{O}_{2}^{-}$} & \multicolumn{2}{|c|}{ NO } \\
\hline & $-H R P$ & $+\mathrm{HRP}$ & $-H R P$ & $+\mathrm{HRP}$ & -HRP & $+\mathrm{HRP}$ & -HRP & $+\mathrm{HRP}$ \\
\hline DHE & 170 & 1882 & 3802 & 3906 & 1372 & 4241 & 104 & 124 \\
\hline DHR & 424 & 6474 & 3571 & 12327 & 51 & 504 & 1776 & 1726 \\
\hline DCF & 775 & 2768 & 10122 & 23966 & 427 & 1806 & 739 & 4115 \\
\hline APF & 260 & 1030 & 5960 & 6945 & 42 & 62 & 51 & 210 \\
\hline DAF & 226 & 3288 & 805 & 7375 & 104 & 1422 & 2430 & 5434 \\
\hline
\end{tabular}

Fluorogenic interactions between selected fluorescence probes $(5 \mu \mathrm{M})$ and reactive oxygen or nitrogen species generated as defined in the text. Numbers represent the mean fluorescence emission intensity on excitation at 490 to $510 \mathrm{~nm}$. In four replicate determinations, the variation was less than $\pm 3 \%$ of the values shown.

readily detect peroxinitrite ion $\left(\mathrm{ONOO}^{-}\right)$. The lack of response of APF to NO shown in Table 1 indicates that this species is not being produced during release of NO from the diethylamine derivative.

While the studies reported in the table do not provide unambiguous information on fluorescence yields, i.e., fluorescence per mole of ROS or RNS, they do provide a comparison of the relative sensitivity of each probe to a given species, along with information on effects of peroxidases. In an attempt to improve specificity, $\mathrm{Xu}$ et al. ${ }^{7}$ has described a naphthofluorescein derivative that emits fluorescence at $670 \mathrm{~nm}$ on exposure to ${ }^{-} \mathrm{O}_{2}^{-}$. It might be preferable to prepare this agent starting with $2^{\prime}, 7^{\prime}$-difluorofluorescein, since naphthofluoresceins are nonpolar and are difficult to work within aqueous environments. Moreover, fluorescence emission from naphthofluorescein is highly $\mathrm{pH}$ dependent, with a $\mathrm{pKa}$ of $\sim 7.5$. This will complicate fluorescence measurements, especially if the probe accumulates in subcellular regions of low $\mathrm{pH}$.

Maeda et al. ${ }^{8}$ described another potentially useful probe for $\cdot \mathrm{O}_{2}^{-}$with only a minor response to $\cdot \mathrm{OH}$. This reagent is based on a nitrobenzenesulfonyl ester structure that can be cleaved by- $\mathrm{SH}$ reagents. This is noted in the report, but in a critical test, only a $50 \mu \mathrm{M}$ concentration was used; this is perhaps $1 \%$ of the expected intracellular GSH concentration.

These examples illustrate the problems associated with attempts to translate results obtained in cell-free systems into corresponding procedures in cell culture. Other commonly encountered problems may relate to ability of fluorescent probes to penetrate the plasma membrane, spontaneous oxidations, $\mathrm{pH}$ of subcellular compartments, and the presence of fluorogenic enzymes, e.g., peroxidases. We propose that if a fluorogenic ROS or RNS probe cannot clearly delineate among different reactive species in a cell-free system, using such a probe to draw conclusions concerning the appearance of such species in culture system may be unrealistic.

As a further example of difficulties in interpretation of data obtained with fluorescent probes, we reported that the Bc1-2 antagonist HA14-1 promoted the apoptotic response to photodynamic therapy ${ }^{9}$ (PDT). When a report ${ }^{10}$ appeared indicating that HA14-1 could cause the spontaneous production of ROS, we considered that the latter effect might explain, at least in part, the synergistic effect. The ability of HA14-1 to evoke formation of ROS was based on studies ${ }^{10}$ involving DCF. The fluorescence observed when HA14-1 was added to cell cultures was actually derived from a fluorogenic reaction between HA14-1 and serum albumin that mimicked ${ }^{11}$ the excitation and emission properties of DCF.

An additional consideration in the use of fluorescent probes in the context of PDT is illustrated by Fig. 1. Murine leukemia P388 cells were incubated in medium containing $2 \mu \mathrm{M}$ benzoporphyrin derivative (Verteporfin, BPD) for $60 \mathrm{~min}$, with a $5 \mu \mathrm{M}$ concentration of the RNS probe DAF added during the final $30 \mathrm{~min}$. The cells were then resuspended in fresh medium and irradiated at $690 \mathrm{~nm}$ $\left(90 \mathrm{~mJ} / \mathrm{cm}^{2}\right)$, conditions we have found capable of killing $50 \%$ of the cell population. Fluorescence microscopy was used to assess the resulting fluorogenic interactions using 450- to 490-nm excitation and monitoring fluorescence at 500 to 550 or at 500 to $700 \mathrm{~nm}$. Experimental conditions included probe alone [Figs. 1(a)-1(c)], probe + photosensitizer in the dark [Figs. 1(d)-1(f)], and after irradiation [Figs. $1(\mathrm{~g})-1(\mathrm{i})]$.

Images obtained with broadband (500- to 700-nm) acquisition indicated a substantial fluorogenic response when BPD was present, but this occurred whether or not the photosensitized cells were irradiated [compare Figs. 1(e) and 1(h)]. The

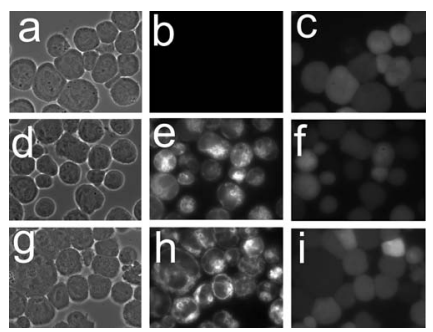

Fig. 1 Phase contrast and fluorescence images of murine leukemia P388 cells incubated with BPD+DAF and irradiated as described in the text: (a) to (c) control cells containing only DAF, (d) to (f) cells containing DAF and BPD but not irradiated, and (g) to (i) irradiated cells loaded with DAF+BPD. Note (a), (d), and (g) are phase contrast images; (b), (e), and (h) are fluorescence images acquired at 500 to $700 \mathrm{~nm}$ for $100 \mathrm{~ms}$; and (c), (f), and (i) are fluorescence at 500 to $550 \mathrm{~nm}$ acquired for $2000 \mathrm{~ms}$. 
fluorescence signal appeared to derive from mitochondria, the site where BPD is localized. ${ }^{12}$ These results illustrate the fact that photosensitizing agents also fluoresce, so that care must be taken to exclude such fluorescence from the probe detection parameters. Fluorescence images obtained with narrowband fluorescence acquisition [Figs. 1(c), 1(f), and 1(i)] revealed that there was no significant fluorogenic response by DAF, hence no significant formation of RNS on irradiation. The relative intensities of the fluorescence can be estimated by the time needed for image acquisition: $100 \mathrm{~ms}$ for Figs. 1(b), 1(e), and 1(h) and $2000 \mathrm{~ms}$ for Figs. 1(c), 1(f), and 1(i).

Based on studies in a cell-free system, together with additional experiments that will be reported elsewhere, we conclude that DHR can be used to distinguish $\mathrm{H}_{2} \mathrm{O}_{2}$ from $\cdot \mathrm{O}_{2}^{-}$, but that peroxidase activity or presence of $\cdot \mathrm{OH}$ can complicate interpretation of results. A prior report had arrived at a similar conclusion. ${ }^{13} \mathrm{DHE}$ is indeed more responsive to $\cdot \mathrm{O}_{2}^{-}$ than to $\mathrm{H}_{2} \mathrm{O}_{2}$, but can be oxidized ${ }^{3}$ by other ROS.

\section{Acknowledgments}

This study was supported by grant CA 23378 from the NCI, NIH. Mr. Price is partially supported by GM058905-11. We thank Ann Marie Santiago for excellent technical assistance.

\section{References}

1. K. Setsukinai, Y. Urano, K. Kakinuma, H. J. Majima, and T. Nagano, "Development of novel fluorescence probes that can reliably detect reactive oxygen species and distinguish specific species," J. Biol. Chem. 278, 3170-3175 (2003).

2. H. Zhao, S. Kalivendi, H. Zhang, J. Joseph, K. Nithipatikom, J. Vásquez-Vivar, and B. Kalyanaraman, "Superoxide reacts with hydroethidine but forms a fluorescent product that is distinctly different from ethidium: potential implications in intracellular fluorescence detection of superoxide," Free Radic Biol. Med. 34, 1359-1368 (2003).
3. J. Zielonka and B. Kalyanaraman, "Hydroethidine- and MitoSOXderived red fluorescence is not a reliable indicator of intracellular superoxide formation: another inconvenient truth," Free Radic Biol. Med. 48, 983-1001 (2010).

4. H. Kohler and H. Jenzer, "Interaction of lactoperoxidase with hydrogen peroxide. Formation of enzyme intermediates and generation of free radicals," Free Radic Biol. Med. 6, 323-339 (1989).

5. H. H. Gorris and D. R. Walt, "Mechanistic aspects of horseradish peroxidase elucidated through single-molecule studies," J. Am. Chem. Soc. 131, 6277-6782 (2009)

6. M. Price, J. J. Reiners Jr., A. M. Santiago, and D. Kessel, "Monitoring singlet oxygen and hydroxyl radical formation with fluorescent probes during photodynamic therapy," Photochem. Photobiol. 85, 1177-1181 (2009).

7. K. Xu, X. Liu, and B. Tang, "Phosphinate-based red fluorescent probe for imaging the superoxide radical anion generated by RAW264.7 macrophages," ChemBioChem 8, 453-458 (2007).

8. H. Maeda, K. Yamamoto, I. Kohno, L. Hafsi, N. Itoh, S. Nakagawa, N. Kanagawa, K. Suzuki, and T. Uno, "Design of a practical fluorescent probe superoxide based on protection-deprotection chemistry of fluoresceine with benzenesulfonyl protecting groups," Chemistry (Weinheim, Ger.) 13, 1946-1954 (2007).

9. D. Kessel, "Promotion of PDT efficacy by a Bcl-2 antagonist," Photochem. Photobiol. 84, 809-814 (2008).

10. J. M. Doshi, D. Tian, and C. Xing, "Ethyl-2-amino-6-bromo-4-(1cyano-2-ethoxy-2-oxoethyl)-4H-chromene-3-carboxylate (HA 14-1), a prototype small-molecule antagonist against antiapoptotic Bcl-2 proteins, decomposes to generate reactive oxygen species that induce apoptosis," Mol. Pharmaceut. 4, 919-928 (2007).

11. D. Kessel, M. Price, and J. J. Reiners Jr., "The Bcl-2 antagonist HA14-1 forms a fluorescent albumin complex that can be mistaken for several oxidized ROS probes," Photochem. Photobiol. 84, 1272 1276 (2008).

12. T. I. Peng, C. J. Chang, M. J. Guo, Y. H. Wang, J. S. Yu, H. Y. Wu, and M. J. Jou, "Mitochondrion-targeted photosensitizer enhances the photodynamic effect-induced mitochondrial dysfunction and apoptosis," Ann. N.Y. Acad. Sci. 1042, 419-428 (2005).

13. L. M. Henderson and J. B. Chappell, "Dihydrorhodamine 123: a fluorescent probe for superoxide generation?" Eur. J. Biochem. 217, $973-$ 980 (1993). 